\title{
Simulation on multidimensional density functions through the Malliavin-Thalmaier formula and its application to finance
}

\author{
Arturo Kohatsu-Higa \\ Graduate School of Engineering Sciences, Osaka University \\ 1-3, Machikaneyama-cho, Toyonaka-shi, Osaka, 560-8531, Japan \\ E-mail: kohatsu@sigmath.es.osaka-u.ac.jp \\ Kazuhiro Yasuda \\ Faculty of Science and Engineering, Hosei University \\ 3-7-2, Kajino-cho, Koganei-shi, Tokyo, 184-8584, Japan \\ E-mail: k_yasuda@hosei.ac.jp
}

\begin{abstract}
The Malliavin-Thalmaier formula was introduced in [4] for use in Monte-Carlo simulation. But when this formula is applied directly for computer simulation, we show that it is unstable. We propose an approximation of the Malliavin-Thalmaier formula. First we prove the central limit theorem to obtain the values of the parameters in Monte-Carlo simulations which achieves a prescribed error level. To prove it, we need the order of the bias and variance of the approximation error. Next we give an idea of the optimal approximation parameter and constants, which is often used in the kernel density estimation method. Finally we apply the MalliavinThalmaier formula and the approximated version to some models in finance and compare their results.
\end{abstract}

\section{Introduction}

We study the problem of estimation of multivariate density functions using Malliavin calculus. That is, let $F=\left(F_{1}, \ldots, F_{d}\right) \in\left(\mathbb{D}^{\infty}\right)^{d}$ be a nondegenerate random vector, where $d \geq 2$. Using the classical integration by parts formula in the Malliavin sense, we have for $\hat{\mathbf{x}}=\left(\hat{x}_{1}, \ldots, \hat{x_{d}}\right) \in \mathbb{R}^{d}$,

$$
p_{F}(\hat{\mathbf{x}})=E\left[\prod_{i=1}^{d} 1_{[0, \infty)}\left(F_{i}-\hat{x}_{i}\right) H_{(1, \ldots, d)}(F ; 1)\right] \text {, }
$$

where $1_{[0, \infty)}(x)$ denotes the indicator function and for $i=2, \ldots, d$,

$$
\begin{aligned}
H_{(1)}(F ; 1) & =\sum_{j=1}^{d} \delta\left(\left(\gamma_{F}^{-1}\right)^{1 j} D F_{j}\right), \\
H_{(1, \ldots, i)}(F ; 1) & =\sum_{j=1}^{d} \delta\left(H_{(1, \ldots, i-1)}(F ; 1)\left(\gamma_{F}^{-1}\right)^{i j} D F_{j}\right),
\end{aligned}
$$

where $\delta$ denotes the adjoint operator of the Malliavin derivative operator $D$ and $\gamma_{F}$ is the Malliavin covariance matrix of $F$. One can find this formula in Nualart [5], Sanz-Solé [6].

In the expression (1), we have to simulate $H_{(1, \ldots, d)}(F ; 1)$, which is given by $d$-iterated Skorohod integral. The Skorohod integral is an extension of the Itô integral to non-adapted integrands. This multiple Skorohod integral is difficult to simulate due to the curse of dimensionality. To solve this problem, Malliavin and Thalmaier [4] introduced a new type of integration by parts formula in the Malliavin sense. Through this formula, they obtained the following new expression for high dimensional probability density functions that can be used in Monte-Carlo simulations;

$$
p_{F}(\hat{\mathrm{x}})=E\left[\sum_{i=1}^{d} \frac{\partial}{\partial x_{i}} Q_{d}(F-\hat{\mathrm{x}}) H_{(i)}(F ; 1)\right],
$$

where $Q_{d}$ is the fundamental solution of the Poisson equation. In the Malliavin-Thalmaier formula, one needs to compute only one Skorohod integral instead of the $d$-iterated Skorohod integrals. Therefore we are free from the curse of dimensionality appearing in formula (1). Nevertheless this formula is unstable when used for computer simulations. That is, its variance is infinity;

$$
E\left[\left(\sum_{i=1}^{d} \frac{\partial}{\partial x_{i}} Q_{d}(F-\hat{\mathrm{x}}) H_{(i)}(F ; 1)\right)^{2}\right]=+\infty .
$$

This situation also appears in kernel density estimation (KDE) type approximations and therefore we propose a similar solution to the present stability problem. Then we give a central limit theorem (CLT) that can be used to determine the optimal bandwidth. And we give an idea of the optimal approximation parameter. 


\section{CLT for approximated Malliavin-Thalmaier formula}

Here, we give the rate of convergence of the modified estimator of the density at $\hat{\mathrm{x}} \in \mathbb{R}^{d}$.

Definitions and Notations

1. For $h>0$ and $\mathrm{x} \in \mathbb{R}^{d}$, define $|\cdot|_{h}$ by

$$
|\mathbf{x}|_{h}:=\sqrt{\sum_{i=1}^{d} x_{i}^{2}+h} .
$$

Without loss of generality, we assume $0<h<1$.

2 . For $i=1, \ldots, d$, define the approximated first derivative of the fundamental solution of Poisson equation; for $\mathrm{x} \in \mathbb{R}^{d}$,

$$
\frac{\partial}{\partial x_{i}} Q_{d}^{h}(\mathrm{x}):=A_{d} \frac{x_{i}}{|\mathrm{x}|_{h}^{d}}
$$

as $A_{d}$ is some known constant.

3. Then we define the approximation to the joint density function of a $d$-dimensional random vector $F=$ $\left(F^{1}, \ldots, F^{d}\right)$ as; for $\hat{\mathrm{x}} \in \mathbb{R}^{d}$,

$$
p_{F}^{h}(\hat{\mathbf{x}}):=E\left[\sum_{i=1}^{d} \frac{\partial}{\partial x_{i}} Q_{d}^{h}(F-\hat{\mathbf{x}}) H_{(i)}(F ; 1)\right] .
$$

This is an approximation of the Malliavin-Thalmaier formula.

Remark 1 For fixed $h>0$, (3) has finite variance;

$$
E\left[\left(\sum_{i=1}^{d} \frac{\partial}{\partial x_{i}} Q_{d}^{h}(F-\hat{\mathbf{x}}) H_{(i)}(F ; 1)\right)^{2}\right]<+\infty .
$$

In what follows $\Rightarrow$ denotes weak convergence and the index $j=1, \ldots, N$ denote $N$ independent copies of the respective random variables.

Theorem 1 Let $Z$ be a random variable with standard normal distribution. And $F^{(j)} \in\left(\mathbb{D}^{\infty}\right)^{d}$ is a random vector which has independent identical distribution.

(i). When $d=2$, set $n=\frac{C}{h \ln \frac{1}{h}}$ and $N=\frac{C^{2}}{h^{2} \ln \frac{1}{h}}$ for some positive constant $C$ fixed throughout.

$$
\begin{aligned}
& n\left(\frac{1}{N} \sum_{j=1}^{N} \sum_{i=1}^{2} \frac{\partial}{\partial x_{i}} Q_{2}^{h}\left(F^{(j)}-\hat{\mathrm{x}}\right) H_{(i)}(F ; 1)^{(j)}-p_{F}(\hat{\mathrm{x}})\right) \\
& \Longrightarrow \sqrt{C_{3}^{\hat{\mathrm{x}}}} Z-C_{1}^{\hat{\mathrm{x}}} C
\end{aligned}
$$

as $h \rightarrow 0$, where $H_{(i)}(F ; 1)^{(j)}, i=1, \ldots, d, j=1, \ldots, N$, denotes the weight obtained in the $j$-th independent simulation (the same that generates $F^{(j)}$ ) and $C_{1}^{\hat{\mathbf{x}}}, C_{3}^{\hat{\mathbf{x}}}$ are some constants.

(ii). When $d \geq 3$, set $n=\frac{C}{h \ln \frac{1}{h}}$ and $N=\frac{C^{2}}{h^{\frac{d}{2}+1}\left(\ln \frac{1}{h}\right)^{2}}$ for some positive constant $C$ fixed throughout.

$$
\begin{aligned}
& n\left(\frac{1}{N} \sum_{j=1}^{N} \sum_{i=1}^{d} \frac{\partial}{\partial x_{i}} Q_{d}^{h}\left(F^{(j)}-\hat{\mathrm{x}}\right) H_{(i)}(F ; 1)^{(j)}-p_{F}(\hat{\mathrm{x}})\right) \\
& \Longrightarrow \sqrt{C_{4}^{\hat{\mathbf{x}}}} Z-C_{1}^{\hat{\mathbf{x}}} C
\end{aligned}
$$

as $h \rightarrow 0$, where $C_{1}^{\hat{x}}, C_{4}^{\hat{x}}$ are some constants.

To prove Theorem 1, we need the order of the error of the approximation to the density.

Proposition 1 Let $F$ be a nondegenerate random vector, then for $\hat{\mathrm{x}}=\left(\hat{x}_{1}, \ldots, \hat{x}_{d}\right) \in \mathbb{R}^{d}$,

$$
p_{F}(\hat{\mathrm{x}})-p_{F}^{h}(\hat{\mathrm{x}})=C_{1}^{\hat{\mathrm{x}}} h \ln \frac{1}{h}+C_{2}^{\hat{\mathrm{x}}} h+o(h),
$$

where $C_{1}^{\hat{\mathrm{x}}}$ and $C_{2}^{\hat{\mathrm{x}}}$ are constants which depend on $\hat{\mathrm{x}}$, but are independent of $h$. The constants can be written explicitly.

Proposition 2 Let $F$ be a nondegenerate random vector. For $\mathrm{x} \in \mathbb{R}^{d}$,

$$
\begin{aligned}
& E\left[\left(\sum_{i=1}^{d} \frac{\partial}{\partial x_{i}} Q_{d}^{h}(F-\hat{\mathbf{x}}) H_{(i)}(F ; 1)-p_{F}(\hat{\mathbf{x}})\right)^{2}\right] \\
& = \begin{cases}C_{3}^{\hat{\mathbf{x}}} \ln \frac{1}{h}+O(1) & d=2, \\
C_{4}^{\hat{\mathbf{x}}} \frac{1}{h^{\frac{d}{2}-1}}+o\left(\frac{1}{h^{\frac{d}{2}-1}}\right) & d \geq 3\end{cases}
\end{aligned}
$$

where $C_{3}^{\hat{\mathrm{x}}}$ and $C_{4}^{\hat{\mathrm{x}}}$ are constants which depend on $\hat{\mathrm{x}}$, but is independent of $h$. The constants can be written explicitly.

The proofs can be found in Kohatsu-Higa and Yasuda [3].

\section{Test simulation results for the multivariate Geometric Brownian motion (the Black-Scholes model)}

We consider the following linear stochastic differential equations (SDEs), which is the well known BlackScholes model; for $i=1, \ldots, d$,

$$
d S_{t}^{i}=\mu_{i} S_{t}^{i} d t+\sum_{j=1}^{d} \sigma_{j}^{i} S_{t}^{i} d W_{t}^{j}
$$

where $\mu_{i}$ and $\sigma_{j}^{i}, i, j=1, \ldots, d$, are positive constants and $W_{t}^{i}, i=1, \ldots, d$, is a standard $d$-dimensional Wiener process.

As we well know, the solution of the SDEs has the lognormal density. Now we compare simulation results 
of the density in four methods when $d=2$, the classical formula in Malliavin Calculus, (1), the MalliavinThalmaier formula, (2), the approximated MalliavinThalmaier formula, (3), and the KDE method (two ways) with the Gaussian kernel and a diagonal bandwidth matrix, which is a nonparametric density estimation method (for more details, see Wand and Jones [7]). Here we use the following parameter values;

$$
\begin{aligned}
& \frac{d S_{t}^{1}}{S_{t}^{1}}=0.01 d t+0.1 d W_{t}^{1}+0.2 d W_{t}^{2} \\
& \frac{d S_{t}^{2}}{S_{t}^{2}}=0.02 d t+0.3 d W_{t}^{1}+0.2 d W_{t}^{2} .
\end{aligned}
$$

We have used the Euler-Maruyama approximation with 10 time steps and $N=10^{4}$ Monte Carlo simulations at each point. The range of the values for $\left(S_{t}^{1}, S_{t}^{2}\right)$ is $[0,200] \times[0,200]$ in the figures (a), and the range of values in figures $(b)$ is $[72.5,82.5] \times[82.5,92.5]$. The initial values are $S_{0}^{1}=S_{0}^{2}=100$ and $t=1$. From Figure 1, we can conclude that the usual method does not work well for both cases. As it can be seen from Figure 2, there are some points where the estimate is unstable. This is clearly due to the infinite variance of the Malliavin-Thalmaier estimator. From Figure 3, we find that we can improve the simulation at the unstable points in Figure 2. Here the choice of $h=0.01$ was an ad-hoc choice. We give the simulation of KDE with global bandwidth in Figure 4 and pointwise bandwidth in Figure 5. For more details about KDE, see Wand and Jones [7].

\section{Estimation of the Optimal Value of $h$}

\subsection{About Optimal $h$}

In this section, we introduce an ad-hoc method to compute the "optimal" $h$ using similar ideas as in kernel density estimation and the central limit theorem obtained in Theorem 1. We consider the following value;

$$
\begin{array}{r}
E\left[\left\{\frac{1}{N} \sum_{j=1}^{N}\left(\sum_{i=1}^{d} \frac{\partial}{\partial x_{i}} Q_{d}^{h}\left(F^{(j)}-\hat{\mathrm{x}}\right) H_{(i)}(F ; 1)^{(j)}\right)\right.\right. \\
\left.\left.-p_{F}(\hat{\mathrm{x}})\right\}^{2}\right] .
\end{array}
$$

From Proposition 1 and Proposition 2, the value is, if $d=2$,

$$
\begin{aligned}
(4)= & \frac{1}{N}\left\{C_{3}^{\hat{\mathbf{x}}} \ln \frac{1}{h}+O(1)\right\} \\
& +\left(1-\frac{1}{N}\right)\left\{C_{1}^{\hat{\mathbf{x}}} h \ln \frac{1}{h}+C_{2}^{\hat{\mathbf{x}}} h+o(h)\right\}^{2} \\
& +\frac{2}{N}\left\{C_{1}^{\hat{\mathbf{x}}} h \ln \frac{1}{h}+C_{2}^{\hat{\mathbf{x}}} h+o(h)\right\} p_{F}(\hat{\mathrm{x}}) \\
& -\frac{1}{N} p_{F}(\hat{\mathrm{x}})^{2}
\end{aligned}
$$

and if $d \geq 3$,

$$
\begin{aligned}
(4)= & \frac{1}{N}\left\{C_{4}^{\hat{\mathbf{x}}} \frac{1}{h^{\frac{d}{2}-1}}+o\left(\frac{1}{h^{\frac{d}{2}-1}}\right)\right\} \\
& +\left(1-\frac{1}{N}\right)\left\{C_{1}^{\hat{\mathbf{x}}} h \ln \frac{1}{h}+C_{2}^{\hat{\mathbf{x}}} h+o(h)\right\}^{2} \\
& +\frac{2}{N}\left\{C_{1}^{\hat{\mathbf{x}}} h \ln \frac{1}{h}+C_{2}^{\hat{\mathbf{x}}} h+o(h)\right\} p_{F}(\hat{\mathbf{x}}) \\
& -\frac{1}{N} p_{F}(\hat{\mathbf{x}})^{2} .
\end{aligned}
$$

Then, from the variance-bias trade-off relationship (see pp.22 in [7]), we select the following terms from the above equation;

$$
g(h):= \begin{cases}\frac{1}{N} C_{3}^{\hat{\mathbf{x}}} \ln \frac{1}{h}+\left(C_{1}^{\hat{\mathbf{x}}}\right)^{2} h^{2}, & d=2, \\ \frac{1}{N h^{\frac{d}{2}-1}} C_{4}^{\hat{\mathbf{x}}}+\left(C_{1}^{\hat{\mathbf{x}}}\right)^{2} h^{2}, & d \geq 3 .\end{cases}
$$

By considering the minimum value of $g(h)$, finally we obtain the following optimal $h$;

$$
h= \begin{cases}\sqrt{\frac{C_{3}^{\hat{\mathbf{x}}}}{2 N\left(C_{1}^{\hat{\mathbf{x}}}\right)^{2}}}, & d=2, \\ \left\{\frac{d-2}{4 N} \frac{C_{4}^{\hat{\mathbf{x}}}}{\left(C_{1}^{\hat{x}}\right)^{2}}\right\}^{\frac{2}{2+d}}, & d \geq 3 .\end{cases}
$$

\subsection{Calculation of Constants $C_{1}^{\mathrm{x}}, C_{3}^{\mathrm{x}}$ and $C_{4}^{\mathrm{x}}$}

Here we give an idea of how to obtain the constants $C_{i}^{\hat{x}}$ for $i=1,3,4$. From our CLT result, we have

$$
\begin{aligned}
& n\left(\frac{1}{N} \sum_{j=1}^{N} \sum_{i=1}^{d} \frac{\partial}{\partial x_{i}} Q_{d}^{h}\left(F^{(j)}-\hat{\mathbf{x}}\right) H_{(i)}(F ; 1)^{(j)}-p_{F}(\hat{\mathbf{x}})\right) \\
& \Longrightarrow \sqrt{C_{a}^{\hat{\mathbf{x}}}} Z-C_{1}^{\hat{\mathbf{x}}},
\end{aligned}
$$

where $C_{a}^{\hat{\mathbf{x}}}=C_{3}^{\hat{\mathbf{x}}}$ if $d=2$ and $C_{a}^{\hat{\mathbf{x}}}=C_{4}^{\hat{\mathbf{x}}}$ if $d \geq 3$. Let $Y_{\hat{\mathrm{x}}}^{h, N}$ be the left hand side of (5). Therefore we consider as follows;

$$
Y_{\hat{\mathrm{x}}}^{h, N} \approx \sqrt{C_{a}^{\hat{\mathrm{x}}}} Z-C_{1}^{\hat{\mathrm{x}}} C .
$$

Then from the above relation, we have the following approximations;

$$
\begin{gathered}
E\left[Y_{\hat{\mathbf{x}}}^{h, N}\right] \approx E\left[\sqrt{C_{a}^{\hat{\mathbf{x}}}} Z-C_{1}^{\hat{\mathbf{x}}} C\right]=-C_{1}^{\hat{\mathbf{x}}} C \\
E\left[\left(Y_{\hat{\mathbf{x}}}^{h, N}\right)^{2}\right] \approx E\left[\left(\sqrt{C_{a}^{\hat{\mathbf{x}}}} Z-C_{1}^{\hat{\mathbf{x}}} C\right)^{2}\right] \\
=C_{a}^{\hat{\mathbf{x}}}+\left(C_{1}^{\hat{\mathbf{x}}} C\right)^{2} .
\end{gathered}
$$

Note that $Z$ is a random variable with the standard normal distribution. And once we determine $h, N$ for test simulation, the constant $C$ is decided by using the relation in the CLT (Theorem 1). In test simulation, the constant $C$ depends on $h, N$. 
We apply Monte-Carlo simulation to the left hand side of the approximations (6) and (7). We obtain a stable result for $C_{a}^{\hat{\mathrm{x}}}$, but the result of $C_{1}^{\hat{\mathrm{x}}}$ is unstable. So we consider another idea of approximation for $C_{1}^{\hat{x}}$, that is,

$$
\frac{1}{M} \sum_{k=1}^{M} Y_{\hat{\mathbf{x}},(k)}^{h, N} \approx \sqrt{C_{a}^{\hat{\mathrm{x}}}} \frac{1}{\sqrt{M}} \tilde{Z}-C_{1}^{\hat{\mathrm{x}}} C^{h, N},
$$

where let $\tilde{Z}$ be a random variable with the standard normal distribution. Now if we try this test simulation $L$ times using different $h$, then we have

$$
\begin{aligned}
\frac{1}{L} \sum_{l=1}^{L}\left(\frac{1}{M} \sum_{k=1}^{M} Y_{\hat{\mathbf{x}},(k)}^{h(l), N}\right) & \approx-\frac{1}{L} \sum_{l=1}^{L} C_{1}^{\dot{\hat{x}}} C^{h(l), N} \\
& =-C_{1}^{\hat{\mathbf{x}}} \frac{1}{L} \sum_{l=1}^{L} C^{h(l), N} .
\end{aligned}
$$

Therefore we obtain $C_{1}^{\hat{\mathbf{x}}}$ as follow;

$$
C_{1}^{\hat{\mathbf{x}}} \approx-\frac{\sum_{l=1}^{L}\left(\frac{1}{M} \sum_{k=1}^{M} Y_{\hat{\mathrm{x}},(k)}^{h(l), N}\right)}{\sum_{l=1}^{L} C^{h(l), N}} .
$$

Remark 2 Once we obtain the constant $C_{1}^{\hat{\mathbf{x}}}$, we can modify the approximation as follows;

$$
\dot{p}_{F}^{h}(\hat{\mathrm{x}})=p_{F}^{h}(\hat{\mathrm{x}})+C_{1}^{\hat{\mathbf{x}}} h \ln \frac{1}{h} .
$$

Then from Proposition 1, we can improve the bias of the error;

$$
\begin{aligned}
p_{F}(\hat{\mathrm{x}})-\dot{p}_{F}^{h}(\hat{\mathrm{x}}) & =p_{F}(\hat{\mathbf{x}})-\left(p_{F}^{h}(\hat{\mathbf{x}})+C_{1}^{\dot{\mathbf{x}}} h \ln \frac{1}{h}\right) \\
& =C_{2}^{\hat{\mathbf{x}}} h+o(h) .
\end{aligned}
$$

\section{Simulation of the Heston model}

Let $S_{t}$ be a stock price process and $v_{t}$ be a volatility process in the Heston model [2];

$$
\begin{aligned}
d S_{t} & =\mu S_{t} d t+\sqrt{v_{t}} S_{t}\left\{\rho d W_{t}^{(2)}+\sqrt{1-\rho^{2}} d W_{t}^{(1)}\right\} \\
d v_{t} & =\gamma\left(\theta-v_{t}\right) d t+\kappa \sqrt{v_{t}} d W_{t}^{(2)}
\end{aligned}
$$

where $\mu, \gamma, \theta, \kappa$ are positive constants satisfying $\gamma \theta \geq$ $\frac{3 \gamma^{2}}{4}, \rho \in[-1,1]$ is a constant and $W^{(1)}$ and $W_{t}^{(2)}$ are Brownian motions and independent of each other. And we transform the model as follows; set $X_{t}:=$ $\ln \left(S_{t} / S_{0}\right)-\mu t$ and $u_{t}:=a v_{t}$.

$$
\begin{aligned}
d X_{t} & =-\frac{u_{t}}{2 a} d t+\sqrt{\frac{u_{t}}{a}}\left\{\sqrt{1-\rho^{2}} d W_{t}^{(1)}+\rho d W_{t}^{(2)}\right\}, \\
d u_{t} & =-\gamma\left(u_{t}-a \theta\right) d t+\sqrt{a} \kappa \sqrt{u_{t}} d W_{t}^{(2)} .
\end{aligned}
$$

We simulate the joint density of $\left(X_{t}, u_{t}\right)$ by using pramater values in Figure 6. We compare values of it in three approaches, through the forward Kolmogorov equation (partial differential equation approach; see Drăgulescu and Yakovenko [1]), the Malliavin-Thalmaier formula and the approximated Malliavin-Thalmaier formula with the optimal $h$, in Figure 7 by using values in Figure 6 . And we give the confidence intervals. Then results of the Kolmogorov equation through finite difference method depend on an approximation of the initial condition (the Dirac delta function) and sizes of difference. Even if we do enough number of Monte-Carlo simulation, we have some singular points in the Malliavin-Thalmaier formula. But we can improve its approximation by using the proposed scheme.

\section{Conclusion}

We give a stable approximation of the MalliavinThalmaier formula. Then we prove the CLT for the approximation error. To prove the CLT, we estimate errors of the bias and variance. And for computer simulation, we give an idea how to choose the optimal approximation parameter and constants, which is often used to obtain the optimal bandwidth in KDE. Finally, we apply the approximated formula for the multiasset Black-Scholes model and the Heston model in finance.

\section{References}

[1] A.A. Drăgulescu and V.M. Yakovenko: Probability distribution of returns in the Heston model with stochastic volatility, Quant. Finance 2, no. 6, pp.443-453, 2002.

[2] S.L. Heston: A closed-form solution for options with stochastic volatility with applications to bond and currency options, The Review of Financial Studies, Vol. 6, No. 2, pp.327-343, 1993.

[3] A. Kohatsu-Higa and K. Yasuda: Estimating Multidimensional Density Functions using the Malliavin-Thalmaier Formula, to appear in SIAM J. Numerical Analysis.

[4] P. Malliavin and A. Thalmaier: Stochastic calculus of variations in mathematical finance, Springer Finance, Springer-Verlag, Berlin, 2006.

[5] D. Nualart: The Malliavin calculus and related topics (Second edition), Probability and its Applications (New York), Springer-Verlag, Berlin 2006.

[6] M. Sanz-Solé: Malliavin Calculus with applications to stochastic partial differential equations, EPFL Press, 2005.

[7] M. P. Wand and M. C. Jones: Kernel smoothing, Monographs on Statistics and Applied Probability, 60, Chapman and Hall, Ltd, London, 1995. 


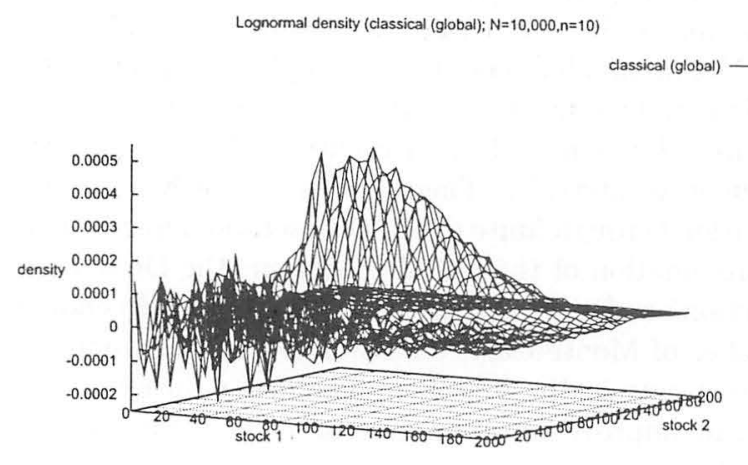

(a) Global view

(b) Local view

Fig. 1: The classical formula (1)

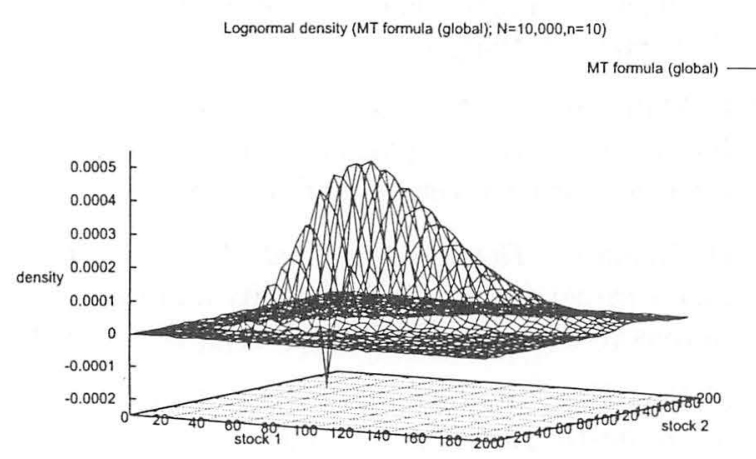

(a) Global view

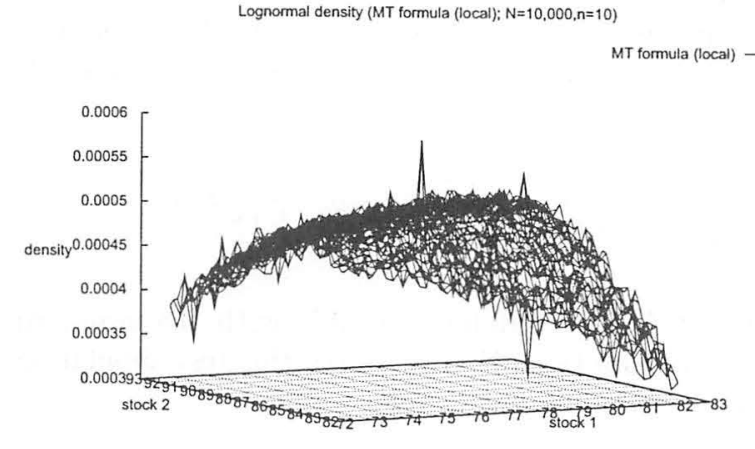

(b) Local view

Fig. 2: The Malliavin-Thalmaier formula (2)

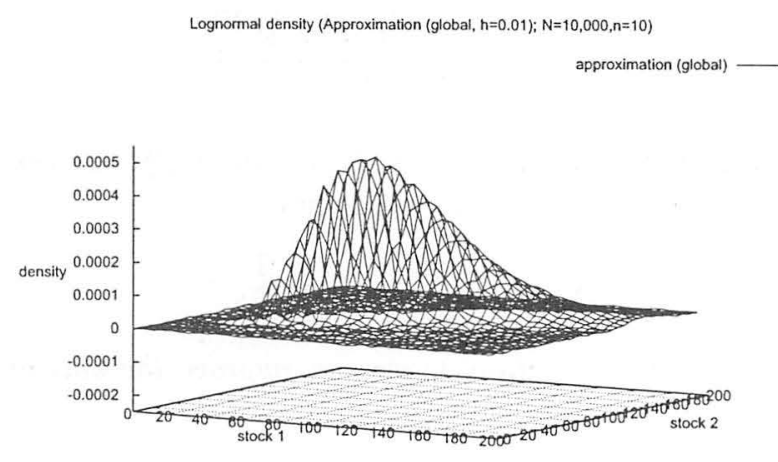

(a) Global view

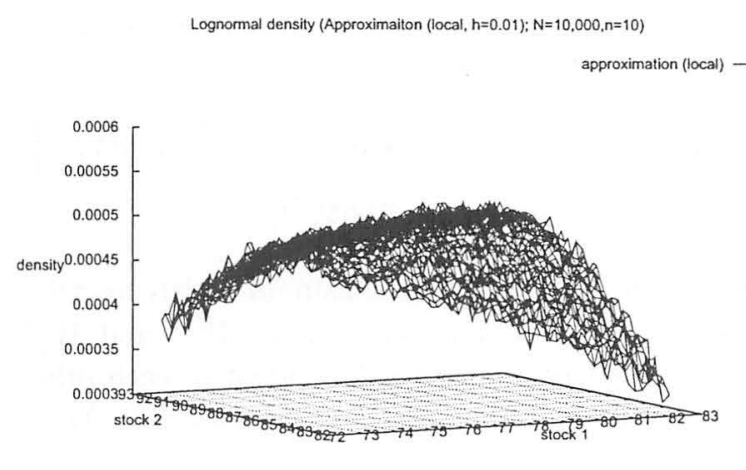

(b) Local view

Fig. 3: The approximation of the Malliavin-Thalmaier formula (3) $(h=0.01)$ 


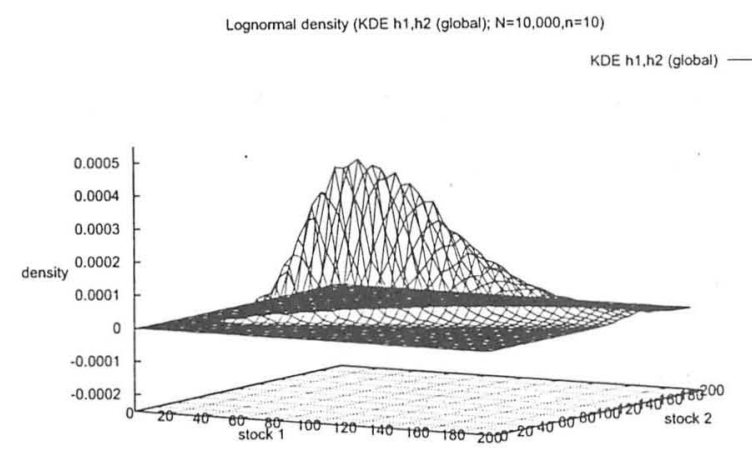

(a) Global view

Lognomal density (KDE h1, h2 (local): N=10,000,n=10)

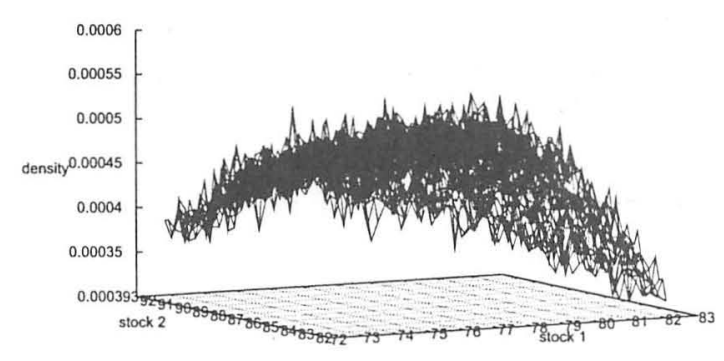

(b) Local view

Fig. 4: KDE with global $h_{1}, h_{2}$

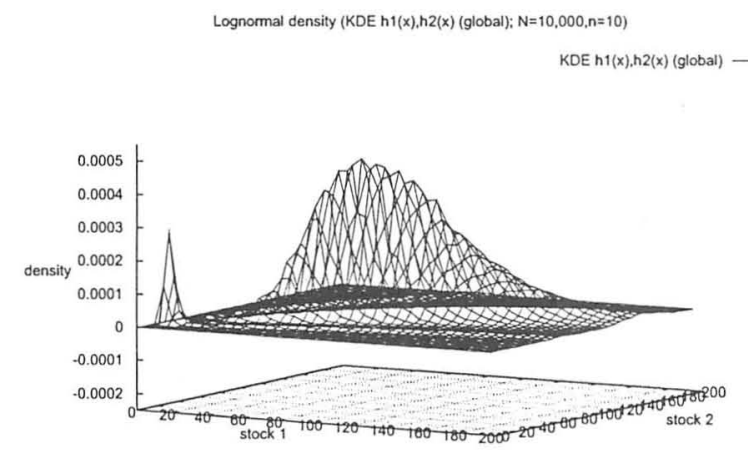

(a) Global view

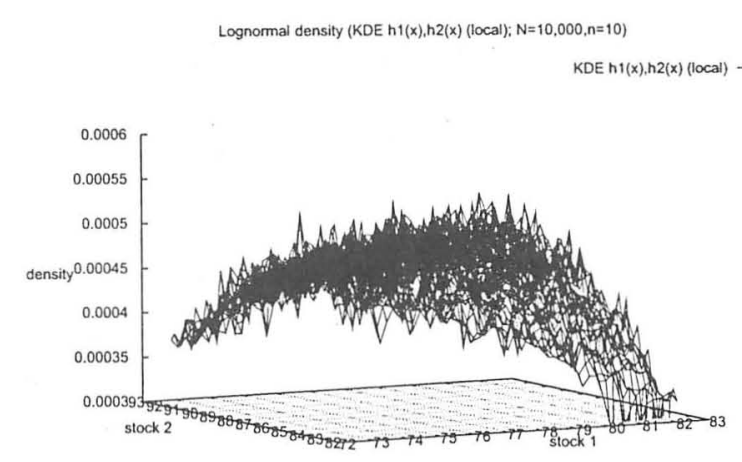

(b) Local view

Fig. 5: KDE with pointwise $h_{1}(\mathrm{x}), h_{2}(\mathrm{x})$

\begin{tabular}{c|c|c} 
Meaning & & Value \\
\hline \hline Initial stock price & $S_{0}$ & 100 \\
\hline Initial vola. value & $v_{0}$ & 0.1 \\
\hline Scale parameter & $a$ & 3 \\
\hline Drift in vola. proc. & $\mu$ & 0.1 \\
\hline Speed of mean reversion & $\gamma$ & 2 \\
\hline Long term mean & $\theta$ & 0.1 \\
\hline Vola. of vola. & $\kappa$ & 0.2 \\
\hline Correlation & $\rho$ & -0.1 \\
\hline Maturity & $t$ & 1 \\
\hline Point of estimation & $X_{t}$ & 0 \\
& $u_{t}$ & 0.3 \\
\hline
\end{tabular}

Fig. 6: Parameters

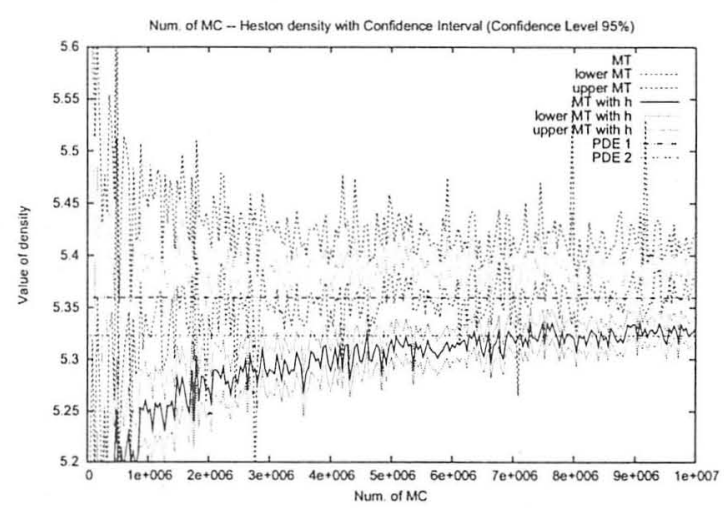

Fig. 7: Values of the transformed Heston model density 